\title{
MINIMAL HAUSDORFF AND \\ MAXIMAL COMPACT SPACES
}

N. SMYTHE and C. A. WILKINS

(received 2 April 1962, revised 25 April, 1962)

\section{Introduction}

Given two topologies $\mathscr{T}_{1}, \mathscr{T}_{2}$ on a set $X, \mathscr{T}_{1}$ is said to be coarser than $\mathscr{T}_{2}$, written $\mathscr{T}_{1} \leqq \mathscr{T}_{2}$, if every set open under $\mathscr{T}_{1}$ is open under $\mathscr{T}_{2}$. A minimal Hausdorff space is then one for which there is no coarser Hausdorff topology etc. Vaidyanathaswamy [4] showed that every compact Hausdorff space is both maximal compact and minimal Hausdorff. This raised the question of whether there exist minimal Hausdorff non-compact spaces and/or maximal compact non-Hausdorff spaces. These questions were in fact answered in the affirmative by Ramanathan [2], Balachandran [1], and Hing Tong [3]. Their examples were, however, all on countable sets, and the topology constructed to answer one question bore no relation to the topology answering the second. In particular, the minimal Hausdorff non-compact topologies were not finer than any maximal compact topology.

We shall here construct two topologies on an extension of the real line (formed by adjoining two points "at infinity") one of which is maximal compact non-Hausdorff, and which is strictly coarser than the other which is minimal Hausdorff non-compact. (In fact there are topologies lying between them.)

Further it is easy to show that there exist minimal $T_{0}$ topologies which are not compact (although every maximal compact space is $T_{0}$ !); and the unique minimum $T_{1}$ topology is always compact; what then may be said of minimal $T_{3}$ and minimal $T_{4}$ spaces? It is shown that every minimal $T_{4}$ space, and every normal minimal $T_{3}$ space is compact; the general question for minimal $T_{3}$ spaces remains open.

The authors would like to acknowledge the helpful suggestions of the referee, especially as regards the material of section 3.

1. Let $R$ be the set of real numbers. Let $E=R \cup\{a\} \cup\{b\}$ with topology $\mathscr{T}$ defined by the following neighbourhood systems:

$$
\begin{aligned}
& \mathscr{W}(x)=\{V \subset E: V \supset(x-d, x+d) \text { for some } d>0\}, \text { if } x \in R \\
& \mathscr{W}(a)=\left\{V \subset E: V \supset\{a\} \cup \bigcup_{r=N}^{\infty}(2 r, 2 r+1) \cup \bigcup_{r=-N}^{-\infty}(2 r, 2 r+1),\right.
\end{aligned}
$$

for some integer $N\}$ 
$\mathscr{W}(b)=\left\{V \subset E: V \supset\{b\} \cup \bigcup_{r=N}^{\infty}(2 r-1,2 r) \cup \bigcup_{r=-N}^{-\infty}(2 r-1,2 r)\right.$,

for some integer $N\}$.

Clearly $E$ is Hausdorff and it induces the usual real line topology on $R$. $E$ is not $T_{3}$ (regular) under $\mathscr{T}$, so it is not compact. We show that $\mathscr{T}$ is minimal Hausdorff.

Suppose $\mathscr{T}^{\prime}$ is a coarser Hausdorff topology on $E$. It is to be proved that every interval $(c, d)$ of $R$ is open in $\mathscr{T}^{\prime}$, and that every basic neighbourhood of $a$ in $\mathscr{T}$ is a neighbourhood of $a$ in $\mathscr{T}^{\prime}$; by symmetry the latter will hold also for $b$, and thus $\mathscr{T}=\mathscr{T}^{\prime}$.

The closed interval $[m, n]$ is compact in $\mathscr{T}$, so it is compact in the coarser topology $\mathscr{T}^{\prime}$, for every open cover of $[m, n]$ in $\mathscr{T}^{\prime}$ is an open cover of $[m, n]$ in $\mathscr{T}$. But $\mathscr{T}^{\prime}$ induces a Hausdorff topology on $[m, n]$ coarser than that induced by $\mathscr{T}$; and every compact Hausdorff topology is minimal Hausdorff, so that $\mathscr{T}$ and $\mathscr{T}^{\prime}$ induce the same topology on $[m, n]$. Then if $x \in(c, d)$ there are open disjoint sets $U, V, W$ of $\mathscr{T}^{\prime}$ containing $x, a, b$ respectively. It follows that for some integer $N$, the only points of $U$ larger in absolute value than $N$ are integers. But $U$ is open in $\mathscr{T}$ so cannot contain isolated points. Thus $U$ is contained in some bounded interval $[m, n]$, which has the usual topology. $U \cap(c, d)$ is open in $[m, n]$, therefore in $U$, therefore in $E$ under $\mathscr{T}^{\prime}$. Then $(c, d)$ contains a $\mathscr{T}^{\prime}$-neighbourhood of every one of its points, so is open in $\mathscr{T}^{\prime}$.

Let $H$ be the basic neighbourhood of $a$ containing $a$ and all intervals $(2 r, 2 r+1)$ for $|r|>$ some integer $N$. There exist disjoint open sets $U, V$ of $\mathscr{T}^{\prime}$ with $a \in U, b \in V$, so that $U$ contains no points of $(2 r-1,2 r)$ for $|r|$ larger than some integer $M . U$ being open in $\mathscr{T}$, it cannot contain arbitrarily large integers without containing neighbourhoods of them, so $U$ does not meet $[2 r-1,2 r]$ for $|r|>M^{\prime}$. Thus $U-H$ is bounded, say $U-H C[c, d]$. Since $[c, d]$ is compact in $\mathscr{T}^{\prime}$ which is Hausdorff, $[c, d]$ is closed. Then $U \cap(E-[c, d])$ is open in $\mathscr{T}^{\prime}$ and is contained in $H$, so $H$ is a $\mathscr{T}^{\prime}$-neighbourhood of $a$. This completes the proof.

2. We may characterise maximal compact spaces by

THEOREM I. A space $E$ is maximal compact if and only if the compact subsets of $E$ are identical with the closed subsets of $E$.

Proof. Necessity: Suppose $E$ is maximal compact under a topology $\mathscr{T}$. Then every closed set is compact. Assume there is a compact non-closed subset $X$ of $E$. Define a topology $\mathscr{T}^{\prime}$ on $E$ by open sets of the form $\left(0^{\prime} \cap(E-X)\right) \cup 0^{\prime \prime}$ where $0^{\prime}$ and $0^{\prime \prime}$ are any sets open in $\mathscr{T}$. This clearly defines a topology which is finer than $\mathscr{T}$ since $X$ is closed in this topology. Let $\left\{G_{s}: s \in S\right\}$ be any open cover of the space $\left(E, \mathscr{T}^{\prime}\right)$. Put $G_{s}=\left(0_{s}^{\prime} \cap(E-X)\right) \cup 0_{s}^{\prime \prime}$, so that $\left\{0_{s}^{\prime} \cup 0_{s}^{\prime \prime}: s \in S\right\}$ is an open cover of 
$(E, \mathscr{T})$; then there exist $s_{1}, \cdots s_{n}$ such that $E=\bigcup_{i=1}^{n}\left(0_{s_{i}}^{\prime} \cup 0_{s_{i}}^{\prime \prime}\right)$, and $E-X \subset \bigcup_{i=1}^{n}\left(0_{s_{i}}^{\prime} \cap(E-X)\right) \cup 0_{s_{i}}^{\prime \prime} \cdot\left\{0_{s}^{\prime \prime}: s \in S\right\}$ is an open cover of the compact space $\left(X, \mathscr{T}_{X}\right)$, so there exist $s_{n+1}, \cdots s_{N}$ such that $X \subset \bigcup_{i=n+1}^{N} 0_{s_{i}}^{\prime \prime}$. Then $\bigcup_{i=1}^{N} G_{i}=E$ and $\mathscr{T}^{\prime}$ is compact which is impossible since $\mathscr{T}$ is maximal compact. Thus every compact set is closed.

Sufficiency: If every closed set is compact, $E$ is compact under $\mathscr{T}$. Now assume every compact set is closed under $\mathscr{T}$. Let $\mathscr{T}^{\prime}(>\mathscr{T})$ be a compact topology on $E$. There exists a set $X$ which is closed under $\mathscr{T}^{\prime}$ but not under $\mathscr{T} ; X$ is then not compact under $\mathscr{T}$, so there is an open cover of $X$ under $\mathscr{T}$ having no finite subcover. This cover is an open cover of $X$ under $\mathscr{T}^{\prime}$ since $\mathscr{T}^{\prime}>\mathscr{T}$, and thus $X$ is not compact but closed in the compact topology $\mathscr{T}^{\prime}$, which is impossible. It follows that $E$ is maximal compact under the topology $\mathscr{T}$. Q.E.D.

Notice this shows that every Hausdorff compact space is maximal compact, since in a Hausdorff space every compact set is closed and in a compact space every closed set is compact.

We may now coarsen the topology on the set $E$ of the preceding section to give an example of a maximal compact non-Hausdorff space. Define $\mathscr{T}_{1}$ on $E$ by neighbourhoods

$$
\begin{aligned}
& \mathscr{W}_{1}(x)=\{U: U \supset(x-d, x+d) \text { for some } d>0\} \text { if } x \in R, \\
& \mathscr{W}_{1}(b)=\left\{U: U \supset\{b\} \cup \bigcup_{|r|=N}^{\infty}(2 r-1,2 r) \text { for some integer } N\right\} \\
& \mathscr{W}_{1}(a)=\left\{U: U \supset\{a\} \cup \bigcup_{|r| \geq N}\left(2 r-d_{r}, 2 r+1+d_{r}\right)\right.
\end{aligned}
$$

for some integer $N$, some $d_{r}>0$.

Clearly this defines a compact non-Hausdorff topology which is strictly coarser than the original topology $\mathscr{T}$. Now if $\mathscr{T}_{1}$ is not maximal compact, there is a compact non-closed set $X$. The interval $[-n, n]$ is closed under $\mathscr{T}_{1}$, which induces the usual topology on $[-n, n]$. Thus $X \cap[-n, n]$ is closed in $X$, and is therefore compact. Being a compact subset of $[-n, n]$, it is closed in $[-n, n]$. Then it follows that $\bar{X} \cap[-n, n]=X \cap[-n, n]$ for all $n$, so that $\bar{X} \cap R=X \cap R$. Since $X \neq X$, either $a$ or $b$ is in $\bar{X}-X$.

If $b \in \bar{X}-X, X$ meets an infinite number of intervals $(2 r-1,2 r)$ for integral $r$; we may choose numbers $d_{r}>0$ such that $X$ meets an infinite number of intervals $\left(2 r-1+d_{r}, 2 r-d_{r}\right)$. The collection of open sets consisting of $\{a\} \cup \bigcup_{r=-\infty}^{\infty}\left(2 r-d_{r}, 2 r+1+d_{r+1}\right)$ together with all sets $(-r, r)$ covers $E-\{b\}$ and therefore $X$; and there is no finite subcover of $X$ from this covering in view of the above. This is impossible since $X$ is compact. Thus $b \notin X-X$.

If $a \in X-X, X$ meets an infinite number of closed intervals [2r, 2r+1], $r$ 
integral. For suppose that for $|\boldsymbol{\gamma}|>N, X$ meets no such intervals. Then $R \cap X$ would eventually be contained in intervals $(2 r-1,2 r)$. For such intervals, $X \cap(2 r-1,2 r)=X \cap[2 r-1,2 r]$ which is compact, so we may choose numbers $d_{r}>0$ such that $X \cap(2 r-1,2 r) \subset\left[2 r-1+d_{r}, 2 r-d_{r}\right]$. Clearly these numbers $d_{r}$ define a neighbourhood of $a$ which does not meet $X$, contradicting $a \in \bar{X}$.

Now consider the open set $\{b\} \cup \bigcup_{r=-\infty}^{\infty}(2 r-1,2 r)$ together with all the open sets $(-r, r)$. This is a covering of $E-\{a\}$, therefore of $X$, yet it has no finite subcover of $X$. So again $a \notin \bar{X}-X$. But this contradicts our original assumption, so that every compact set is closed and $\mathscr{T}_{1}$ is maximal compact.

3. It is natural now to ask whether every minimal $T_{3}$ ( $T_{1}$ and regular) space, and/or every minimal $T_{4}\left(T_{1}\right.$ and normal) space is compact. The question is partly answered in

TheOREM 2. Every minimal $T_{4}$ space is compact.

CoRollary. Every normal minimal $T_{3}$ space is compact. In fact, the class of minimal $T_{4}$ spaces is identical with the class of normal minimal $T_{3}$ spaces, and with the class of compact Hausdorff spaces.

Proof. Suppose $X$ is normal and Hausdorff under a topology $\mathscr{T}$, but not compact. We shall construct a strictly coarser $T_{4}$ topology on $X$, thus proving the theorem. The corollary follows immediately.

Since $X$ is not compact there is a filter-base of closed sets of $X$ with empty adherence; i.e., a family $\mathscr{F}$ of closed sets such that any finite intersection of sets of $\mathscr{F}$ is a member of $\mathscr{F}$, and whose intersection is empty while the intersection of every finite subfamily is non-empty. Choose any point $x \in X$ and define a topology $\mathscr{T}^{\prime}$ on $X$ as follows: the open sets of $\mathscr{T}^{\prime}$ are those open sets of $\mathscr{T}$ not containing $x$, together with those open sets of $\mathscr{T}$ containing $x$ and some $F \in \mathscr{F}$. From the finite intersection property of filter-bases it follows that $\mathscr{T}^{\prime}$ is a topology; and $\mathscr{T}^{\prime}$ is strictly coarser than $\mathscr{T}$, for if every set $G$ open in $\mathscr{T}$ containing $x$ contained some set $F \in \mathscr{T}, G$ would meet every $F \in \mathscr{F}$ and it would follow $x \in F=F$, for every $F \in \mathscr{F}$, which is impossible.

The topology $\mathscr{T}^{\prime}$ is $T_{1}$ since for any $y \in X, X-\{y\}$ is open in $\mathscr{T}$ and contains some $F \in \mathscr{F}(y \notin F$ for some $F \in \mathscr{F})$, and so is open in $\mathscr{T}^{\prime}$.

Further the topology $\mathscr{T}^{\prime}$ is normal. For let $A, B$ be two disjoint sets closed in $\mathscr{T}^{\prime}$. Suppose $x \notin A$, since $x$ cannot be in both. $X-A$ is open in $\mathscr{T}$, so contains some $F \in \mathscr{F}$. The sets $A, B \cup F \cup\{x\}$ are disjoint sets closed under $\mathscr{T}$ which is normal, so there exist disjoint sets $U, V$ open in $\mathscr{T}$ such that $A \subset U, B \cup F \cup\{x\} \subset V . x \notin U$ and $F \subset V$ so that $U$ and $V$ are both open in $\mathscr{T}^{\prime}$. This completes the proof, since $A \subset U, B \subset V$. 


\section{References}

[1] Balachandran, V. K., Minimal Bicompact Spaces. J. Ind. Math. Soc., N.S. Vol. 12, pp. $47-48(1948)$.

[2] Ramanathan, A., A Characterisation of Maximal Hausdorff Spaces, J. Ind. Math. Soc., N.S. Vol. 11, pp. 23-80 (1947).

[3] Tong, H., Minimal Bicompact Spaces, Bull. Am. Math Soc.., Vol. 54, pp. $478-479$ (1948).

[4] Vaidyanathaswamy, R., Treatise on Set Topology (Ind. Math. Soc. Madras 1947).

University of New South Wales. 